\title{
Generalization of weighted Ostrowski-Grüss type inequality by using Korkine's identity
}

\author{
Silvestru Sever Dragomir, Nazia Irshad and Asif R. Khan
}

\begin{abstract}
We obtain generalized weighted Ostrowski-Grüss type inequality with parameters for differentiable functions by using the weighted Korkine's identity, and we then apply these obtained inequalities to probability density functions. Also, we discuss some applications of numerical quadrature rules.
\end{abstract}

Mathematics Subject Classification (2010): 26D15, 26D20, 26D99.

Keywords: Weighted Ostrowski-Grüss Inequality, Euclidean norm, Weighted Korkine's identity, Probability density function.

\section{Introduction}

Inequalities are frequently used in different areas of sciences. Inequalities play a major role in numerical analysis for error estimation of bounds. In numerical analysis, inequalities help us to find out the best bounds. In the last few years, the mid-point, trapezoid and Simpson's type rules have been examined with the perspective of getting bounds for the quadrature rules. By using modern theory of inequalities and weighted Peano kernel approach, present article is devoted to investigate several refinements of inequalities for weighted Ostrowski-Grüss type inequality and to deduce explicit bounds for the numerical quadrature rules in terms of variety of norms.

In 1935, Grüss gave a celebrated integral inequality known as Grüss inequality [4] which provides a bound on Čebyšev inequality (see [8], p.297) which establishes a relation between the integral of the product of two functions and the product of the integral of two functions. To highlight its importance, these inequalities are discussed in detail by D. S. Mitrinović, J. E. Pečarić and A. M. Fink in their books "Classical and New Inequalities in Analysis" [8] and "Inequalities involving Functions and their Integrals and Derivatives" [9]. The Grüss inequality is stated as:

Proposition 1.1. Let $\psi, \varphi:\left[b_{0}, b_{1}\right] \rightarrow \mathbb{R}$ be two integrable functions, satisfying the conditions

$$
m \leq \psi(\eta) \leq M, \quad n \leq \varphi(\eta) \leq N
$$


for each $\eta \in\left[b_{0}, b_{1}\right]$, where $m, M, n, N$ are given real constants. Further, let the Čebyšev functional be defined as

$$
T(\psi, \varphi)=\frac{1}{b_{1}-b_{0}} \int_{b_{0}}^{b_{1}} \psi(\eta) \varphi(\eta) d \eta-\frac{1}{\left(b_{1}-b_{0}\right)^{2}} \int_{b_{0}}^{b_{1}} \psi(\eta) d \eta \int_{b_{0}}^{b_{1}} \varphi(\eta) d \eta
$$

Then,

$$
|T(\psi, \varphi)| \leq \frac{1}{4}(M-m)(N-n)
$$

where the constant $\frac{1}{4}$ is the best possible.

In 1997, using the Grüss inequality S. S. Dragomir and S. Wang [3] verified the following Ostrowski-Grüss type integral inequality:

Proposition 1.2. Let $\psi: I \rightarrow \mathbb{R}$, where $I \subseteq \mathbb{R}$ is an interval, be a mapping differentiable in the interior $I^{0}$ of $I$, and let $b_{0}, b_{1} \in \overline{I^{0}}$ with $b_{0}<b_{1}$. If $\nu \leq \psi^{\prime}(\eta) \leq \mu, \eta \in\left[b_{0}, b_{1}\right]$ for some constants $\nu, \mu \in \mathbb{R}$. Then

$$
\begin{array}{r}
\left|\psi(\eta)-\frac{1}{b_{1}-b_{0}} \int_{b_{0}}^{b_{1}} \psi(s) d s-\frac{\psi\left(b_{1}\right)-\psi\left(b_{0}\right)}{b_{1}-b_{0}}\left(\eta-\frac{b_{0}+b_{1}}{2}\right)\right| \\
\leq \frac{1}{4}\left(b_{1}-b_{0}\right)(\mu-\nu)
\end{array}
$$

for all $\eta \in\left[b_{0}, b_{1}\right]$.

Relation (1.1) generates a link between the Ostrowski inequality [10] and the Grüss inequality [8].

In 2000, Proposition 1.2 was improved by M. Matić, J. E. Pečarić and N. Ujević [7].

Proposition 1.3. Let the assumptions of Proposition 1.2 be true. Then

$$
\begin{array}{r}
\left|\psi(\eta)-\frac{1}{b_{1}-b_{0}} \int_{b_{0}}^{b_{1}} \psi(s) d s-\frac{\psi\left(b_{1}\right)-\psi\left(b_{0}\right)}{b_{1}-b_{0}}\left(\eta-\frac{b_{0}+b_{1}}{2}\right)\right| \\
\leq \frac{1}{4 \sqrt{3}}(\mu-\nu)\left(b_{1}-b_{0}\right)
\end{array}
$$

for all $\eta \in\left[b_{0}, b_{1}\right]$.

In the same year, N. S. Barnett, S. S. Dragomir and A. Sofo [2] worked upon inequality (1.2). The improved version of the inequality states that:

Proposition 1.4. Let $\psi: I \rightarrow \mathbb{R}$ be an absolutely continuous function whose derivative $\psi^{\prime} \in L_{2}\left[b_{0}, b_{1}\right]$, if $\nu \leq \psi^{\prime}(\eta) \leq \mu, \eta \in\left[b_{0}, b_{1}\right]$ for some constants $\nu, \mu \in \mathbb{R}$. Then

$$
\begin{array}{r}
\left|\psi(\eta)-\frac{1}{b_{1}-b_{0}} \int_{b_{0}}^{b_{1}} \psi(s) d s-\frac{\psi\left(b_{1}\right)-\psi\left(b_{0}\right)}{b_{1}-b_{0}}\left(\eta-\frac{b_{0}+b_{1}}{2}\right)\right| \\
\leq \frac{\left(b_{1}-b_{0}\right)}{2 \sqrt{3}}\left[\frac{1}{b_{1}-b_{0}}\left\|\psi^{\prime}\right\|_{2}^{2}-\left(\frac{\psi\left(b_{1}\right)-\psi\left(b_{0}\right)}{b_{1}-b_{0}}\right)^{2}\right]^{\frac{1}{2}} \\
\leq \frac{1}{4 \sqrt{3}}(\mu-\nu)\left(b_{1}-b_{0}\right) .
\end{array}
$$


In 2006, B. G. Pachpatte discussed inequalities by using Montgomery's identity. In 2010, F. Zafar and N. A. Mir in [12] introduced some parameters in the Peano kernel, defined as

$$
K(\eta, s)= \begin{cases}s-\left(b_{0}+h \frac{b_{1}-b_{0}}{2}\right), & \text { if } \quad s \in\left[b_{0}, \eta\right] \\ s-\left(b_{1}-h \frac{b_{1}-b_{0}}{2}\right), & \text { if } \quad s \in\left(\eta, b_{1}\right]\end{cases}
$$

and generalized the inequality (1.3) in the next proposition:

Proposition 1.5. Let the assumptions of Proposition 1.4 be valid. Then the inequality of Ostrowski-Grüss type is

$$
\begin{aligned}
& \mid(1-h)\left[\psi(\eta)-\frac{\psi\left(b_{1}\right)-\psi\left(b_{0}\right)}{b_{1}-b_{0}}\left(\eta-\frac{b_{0}+b_{1}}{2}\right)\right] \\
& +h \frac{\psi\left(b_{0}\right)+\psi\left(b_{1}\right)}{2}-\frac{1}{b_{1}-b_{0}} \int_{b_{0}}^{b_{1}} \psi(s) d s \mid \\
\leq & {\left[\frac{\left(b_{1}-b_{0}\right)^{2}}{12}\left(3 h^{2}-3 h+1\right)+h(1-h)\left(\eta-\frac{b_{0}+b_{1}}{2}\right)^{2}\right]^{\frac{1}{2}} } \\
& \times\left[\frac{1}{b_{1}-b_{0}}\left\|\psi^{\prime}\right\|_{2}^{2}-\left(\frac{\psi\left(b_{1}\right)-\psi\left(b_{0}\right)}{b_{1}-b_{0}}\right)^{2}\right]^{\frac{1}{2}} \\
\leq & \frac{1}{2}(\mu-\nu)\left[\frac{\left(b_{1}-b_{0}\right)^{2}}{12}\left(3 h^{2}-3 h+1\right)+h(1-h)\left(\eta-\frac{b_{0}+b_{1}}{2}\right)^{2}\right]^{\frac{1}{2}}
\end{aligned}
$$

for all $\eta \in\left[b_{0}-h \frac{b_{1}-b_{0}}{2}, b_{1}+h \frac{b_{1}-b_{0}}{2}\right] \quad$ and $\quad h \in[0,1]$.

In this article, we generalized inequality (1.4) for differentiable functions in terms of weights and parameters. The generalization of Grüss inequality will be established by introducing weighted Peano kernel. The parameters and weights can be adjusted to recapture the previous results. Our first section is based on Introduction and Propositions. In the second section, we would state results related to weighted OstrowskiGrüss inequality by using the technique of weighted Korkine's identity. In the third section, we apply our established results to probability density functions. Fourth section is based on applications of numerical quadrature rules. Our last section concludes the article. 


\section{Main result}

We need following two lemmas from [1] to prove our main result.

Lemma 2.1. (Weighted Korkine's Identity) Let $p, \psi, \varphi:\left[b_{0}, b_{1}\right] \rightarrow \mathbb{R}$ be the measurable mapping for which the integrals involved in the following identity exist and finite. Then

$$
\begin{array}{r}
\int_{b_{0}}^{b_{1}} p(s) d s \int_{b_{0}}^{b_{1}} p(s) \psi(s) \varphi(s) d s-\int_{b_{0}}^{b_{1}} p(s) \psi(s) d s \int_{b_{0}}^{b_{1}} p(s) \varphi(s) d s \\
=\frac{1}{2} \int_{b_{0}}^{b_{1}} \int_{b_{0}}^{b_{1}} p(s) p(t)(\psi(s)-\psi(t))(\varphi(s)-\varphi(t)) d s d t .
\end{array}
$$

Lemma 2.2. Let the assumptions of Lemma 2.1 be valid. Then we have the following inequality

$$
0 \leq \int_{b_{0}}^{b_{1}} p(s) \psi^{2}(s) d s-\left(\int_{b_{0}}^{b_{1}} p(s) \psi(s) d s\right)^{2} \leq \frac{1}{4}(M-m)^{2}
$$

where $m \leq \psi(s) \leq M$ a.e. on $\left[b_{0}, b_{1}\right]$.

Throughout the paper $\alpha=b_{0}+h \frac{b_{1}-b_{0}}{2}$ and $\beta=b_{1}-h \frac{b_{1}-b_{0}}{2}$ where $h \in[0,1]$.

Theorem 2.3. Let the assumptions of Proposition 1.4 be valid. Then we get the inequality

$$
\begin{aligned}
& \mid \psi(\eta) \int_{\alpha}^{\beta} p(u) d u+\psi\left(b_{0}\right) \int_{b_{0}}^{\alpha} p(u) d u+\psi\left(b_{1}\right) \int_{\beta}^{b_{1}} p(u) d u \\
& -\left(\eta \int_{\alpha}^{\beta} p(u) d u+b_{0} \int_{b_{0}}^{\alpha} p(u) d u+b_{0} \int_{\beta}^{b_{1}} p(u) d u-\int_{b_{0}}^{b_{1}} p(s) s d s\right) \\
& \left.\quad \times\left(\int_{b_{0}}^{b_{1}} p(s) \psi^{\prime}(s) d s\right)-\left.\int_{b_{0}}^{b_{1}} p(s) \psi(s) d s\right|^{2}\right]^{\frac{1}{2}} \\
& \leq\left[\int_{b_{0}}^{b_{1}} \frac{K_{p}^{2}(\eta, s) d s}{p(s)}-\left(\int_{b_{0}}^{b_{1}} K_{p}(\eta, s) d s\right)^{\frac{1}{2}}\right. \\
& \quad \times\left[\int_{b_{0}}^{b_{1}} p(s)\left[\psi^{\prime}(s)\right]^{2} d s-\left(\int_{b_{0}}^{b_{1}} p(s) \psi^{\prime}(s) d s\right)^{\frac{1}{2}}\right]^{2} \\
& \leq \frac{1}{2}(\mu-\nu) H_{p}(\eta, s)
\end{aligned}
$$

where

$$
H_{p}(\eta, s)=\left[\int_{b_{0}}^{b_{1}} \frac{K_{p}^{2}(\eta, s) d s}{p(s)}-\left(\int_{b_{0}}^{b_{1}} K_{p}(\eta, s) d s\right)^{2}\right]^{\frac{1}{2}}
$$


and $p:\left[b_{0}, b_{1}\right] \rightarrow[0, \infty)$ is some probability density function satisfying

$$
\int_{b_{0}}^{b_{1}} p(s) d s=1
$$

for all $\eta \in[\alpha, \beta] \quad$ and $\quad h \in[0,1]$.

Proof. We have the kernel as defined in $[5], K_{p}(\eta, s):\left[b_{0}, b_{1}\right]^{2} \rightarrow \mathbb{R}$

$$
K_{p}(\eta, s)=\left\{\begin{array}{l}
\int_{\alpha}^{s} p(u) d u, \quad \text { if } \quad s \in\left[b_{0}, \eta\right], \\
\int_{\beta}^{s} p(u) d u, \quad \text { if } \quad s \in\left(\eta, b_{1}\right] .
\end{array}\right.
$$

From (2.1), we get the Korkine's identity in the form of

$$
\begin{aligned}
& \int_{b_{0}}^{b_{1}} K_{p}(\eta, s) \psi^{\prime}(s) d s-\int_{b_{0}}^{b_{1}} K_{p}(\eta, s) d s \int_{b_{0}}^{b_{1}} p(s) \psi^{\prime}(s) d s \\
& =\frac{1}{2} \int_{b_{0}}^{b_{1}} \int_{b_{0}}^{b_{1}} p(s) p(t)\left(\frac{K_{p}(\eta, s)}{p(s)}-\frac{K_{p}(\eta, t)}{p(t)}\right)\left(\psi^{\prime}(s)-\psi^{\prime}(t)\right) d s d t .
\end{aligned}
$$

From [5], we have

$$
\begin{aligned}
\int_{b_{0}}^{b_{1}} K_{p}(\eta, s) \psi^{\prime}(s) d s= & \psi(\eta) \int_{\alpha}^{\beta} p(u) d u+\psi\left(b_{0}\right) \int_{b_{0}}^{\alpha} p(u) d u \\
& +\psi\left(b_{1}\right) \int_{\beta}^{b_{1}} p(u) d u-\int_{b_{0}}^{b_{1}} p(s) \psi(s) d s
\end{aligned}
$$

and

$$
\begin{aligned}
\int_{b_{0}}^{b_{1}} K_{p}(\eta, s) d s= & \eta \int_{\alpha}^{\beta} p(u) d u+b_{0} \int_{b_{0}}^{\alpha} p(u) d u \\
& +b_{1} \int_{\beta}^{b_{1}} p(u) d u-\int_{b_{0}}^{b_{1}} p(s) s d s .
\end{aligned}
$$

By putting (2.5) and (2.6) in (2.4), we get

$$
\begin{aligned}
& \psi(\eta) \int_{\alpha}^{\beta} p(u) d u+\psi\left(b_{0}\right) \int_{b_{0}}^{\alpha} p(u) d u+\psi\left(b_{1}\right) \int_{\beta}^{b_{1}} p(u) d u \\
& -\left(\eta \int_{\alpha}^{\beta} p(u) d u+b_{0} \int_{b_{0}}^{\alpha} p(u) d u+b_{1} \int_{\beta}^{b_{1}} p(u) d u-\int_{b_{0}}^{b_{1}} p(s) s d s\right) \\
& \times\left(\int_{b_{0}}^{b_{1}} p(s) \psi^{\prime}(s) d s\right)-\int_{b_{0}}^{b_{1}} p(s) \psi(s) d s \\
& =\frac{1}{2} \int_{b_{0}}^{b_{1}} \int_{b_{0}}^{b_{1}} p(s) p(t)\left(\frac{K_{p}(\eta, s)}{p(s)}-\frac{K_{p}(\eta, t)}{p(t)}\right)\left(\psi^{\prime}(s)-\psi^{\prime}(t)\right) d s d t
\end{aligned}
$$

$\forall \eta \in[\alpha, \beta]$ 
Using Cauchy-Schwartz inequality for double integrals, we get

$$
\begin{aligned}
& \left|\frac{1}{2} \int_{b_{0}}^{b_{1}} \int_{b_{0}}^{b_{1}} p(s) p(t)\left(\frac{K_{p}(\eta, s)}{p(s)}-\frac{K_{p}(\eta, t)}{p(t)}\right)\left(\psi^{\prime}(s)-\psi^{\prime}(t)\right) d s d t\right| \\
\leq & \left(\frac{1}{2} \int_{b_{0}}^{b_{1}} \int_{b_{0}}^{b_{1}} p(s) p(t)\left(\frac{K_{p}(\eta, s)}{p(s)}-\frac{K_{p}(\eta, t)}{p(t)}\right)^{2} d s d t\right)^{\frac{1}{2}} \\
& \times\left(\frac{1}{2} \int_{b_{0}}^{b_{1}} \int_{b_{0}}^{b_{1}} p(s) p(t)\left(\psi^{\prime}(s)-\psi^{\prime}(t)\right)^{2} d s d t\right)^{\frac{1}{2}} .
\end{aligned}
$$

By using (2.4), we get the following identities

$$
\begin{aligned}
\frac{1}{2} \int_{b_{0}}^{b_{1}} \int_{b_{0}}^{b_{1}} p(s) p(t)\left(\frac{K_{p}(\eta, s)}{p(s)}-\frac{K_{p}(\eta, t)}{p(t)}\right)^{2} d s d t & \\
& =\int_{b_{0}}^{b_{1}} \frac{K_{p}^{2}(\eta, s) d s}{p(s)}-\left(\int_{b_{0}}^{b_{1}} K_{p}(\eta, s) d s\right)^{2}
\end{aligned}
$$

and

$$
\begin{aligned}
\frac{1}{2} \int_{b_{0}}^{b_{1}} \int_{b_{0}}^{b_{1}} p(s) p(t)\left(\psi^{\prime}(s)-\psi^{\prime}(t)\right)^{2} d s d t & \\
= & \int_{b_{0}}^{b_{1}} p(s)\left[\psi^{\prime}(s)\right]^{2} d s-\left(\int_{b_{0}}^{b_{1}} p(s) \psi^{\prime}(s) d s\right)^{2}
\end{aligned}
$$

Using weighted Ostrowski Grüss inequality (2.2), if $\nu \leq \psi^{\prime}(s) \leq \mu$ and $s \in\left(b_{0}, b_{1}\right)$, we get

$$
0 \leq \int_{b_{0}}^{b_{1}} p(s)\left(\psi^{\prime}(s)\right)^{2} d s-\left(\int_{b_{0}}^{b_{1}} p(s) \psi^{\prime}(s) d s\right)^{2} \leq \frac{1}{4}(\mu-\nu)^{2} .
$$

Using $(2.7)-(2.11)$, we obtain

$$
\begin{aligned}
& \mid \psi(\eta) \int_{\alpha}^{\beta} p(u) d u+\psi\left(b_{0}\right) \int_{b_{0}}^{\alpha} p(u) d u+\psi\left(b_{1}\right) \int_{\beta}^{b_{1}} p(u) d u \\
& -\left(\eta \int_{\alpha}^{\beta} p(u) d u+b_{0} \int_{b_{0}}^{\alpha} p(u) d u+b_{1} \int_{\beta}^{b_{1}} p(u) d u-\int_{b_{0}}^{b_{1}} p(s) s d s\right) \\
& \left.\times\left(\int_{b_{0}}^{b_{1}} p(s) \psi^{\prime}(s) d s\right)-\left.\int_{b_{0}}^{b_{1}} p(s) \psi(s) d s\right|^{2}\right]^{\frac{1}{2}} \\
\leq & {\left[\int_{b_{0}}^{b_{1}} \frac{K_{p}^{2}(\eta, s) d s}{p(s)}-\left(\int_{b_{0}}^{b_{1}} K_{p}(\eta, s) d s\right)^{1}\right.}
\end{aligned}
$$




$$
\begin{aligned}
& \times\left[\int_{b_{0}}^{b_{1}} p(s)\left[\psi^{\prime}(s)\right]^{2} d s-\left(\int_{b_{0}}^{b_{1}} p(s) \psi^{\prime}(s) d s\right)^{2}\right]^{\frac{1}{2}} \\
\leq & \frac{1}{2}(\mu-\nu)\left[\int_{b_{0}}^{b_{1}} \frac{K_{p}^{2}(\eta, s) d s}{p(s)}-\left(\int_{b_{0}}^{b_{1}} K_{p}(\eta, s) d s\right)^{2}\right] \\
= & \frac{1}{2}(\mu-\nu) H_{p}(\eta, s)
\end{aligned}
$$

which proves our result (2.3).

We can state some special cases of (2.3).

Remark 2.4. If we put $p(s) \equiv \frac{1}{b_{1}-b_{0}}$ in $(2.3)$, then we get the result (1.4) of [12].

Remark 2.5. If we put $h=0$ in (2.3), then $\alpha=b_{0}$ and $\beta=b_{1}$, then following inequality holds

$$
\begin{aligned}
& \left|\psi(\eta)-\left(\eta-\int_{b_{0}}^{b_{1}} p(s) s d s\right)\left(\int_{b_{0}}^{b_{1}} p(s) \psi^{\prime}(s) d s\right)-\int_{b_{0}}^{b_{1}} p(s) \psi(s) d s\right| \\
\leq & {\left[\int_{b_{0}}^{b_{1}} \frac{K_{p}^{2}(\eta, s) d s}{p(s)}-\left(\int_{b_{0}}^{b_{1}} K_{p}(\eta, s) d s\right)^{2}\right]^{\frac{1}{2}} } \\
& \times\left[\int_{b_{0}}^{b_{1}} p(s)\left[\psi^{\prime}(s)\right]^{2} d s-\left(\int_{b_{0}}^{b_{1}} p(s) \psi^{\prime}(s) d s\right)^{2}\right]^{\frac{1}{2}} \\
\leq & \frac{1}{2}(\mu-\nu) H_{p}(\eta, s) .
\end{aligned}
$$

The above inequality is Theorem 1 of paper [6].

Remark 2.6. If we put $p(s) \equiv \frac{b_{1}-b_{0}}{2}$ in $(2.12)$, then we get the inequality (1.3) of [2].

Remark 2.7. If we put $h=1$ in (2.3), then $\alpha=\beta=\frac{b_{0}+b_{1}}{2}$, then following inequality holds

$$
\begin{aligned}
& \mid \psi\left(b_{0}\right) \int_{b_{0}}^{\frac{b_{0}+b_{1}}{2}} p(u) d u+\psi\left(b_{1}\right) \int_{b_{1}}^{\frac{b_{0}+b_{1}}{2}} p(u) d u-\int_{b_{0}}^{b_{1}} p(s) \psi(s) d s \\
& -\left(b_{0} \int_{b_{0}}^{\frac{b_{0}+b_{1}}{2}} p(u) d u+b_{1} \int_{\frac{b_{0}+b_{1}}{2}}^{b_{1}} p(u) d u-\int_{b_{0}}^{b_{1}} p(s) s d s\right)\left(\int_{b_{0}}^{b_{1}} p(s) \psi^{\prime}(s) d s\right) \mid
\end{aligned}
$$




$$
\begin{aligned}
& \leq\left[\int_{b_{0}}^{b_{1}} \frac{K_{p}^{2}(\eta, s) d s}{p(s)}-\left(\int_{b_{0}}^{b_{1}} K_{p}(\eta, s) d s\right)^{2}\right]^{\frac{1}{2}} \\
& \quad \times\left[\int_{b_{0}}^{b_{1}} p(s)\left[\psi^{\prime}(s)\right]^{2} d s-\left(\int_{b_{0}}^{b_{1}} p(s) \psi^{\prime}(s) d s\right)^{2}\right]^{\frac{1}{2}} \\
& \leq \frac{1}{2}(\mu-\nu) H_{p}(\eta, s) .
\end{aligned}
$$

Remark 2.8. If we put $p(s) \equiv \frac{1}{b_{1}-b_{0}}$ in $(2.13)$, then trapezoidal inequality holds

$$
\begin{aligned}
& \left|\frac{\psi\left(b_{0}\right)+\psi\left(b_{1}\right)}{2}-\frac{1}{b_{1}-b_{0}} \int_{b_{0}}^{b_{1}} \psi(s) d s\right| \\
\leq & \frac{b_{1}-b_{0}}{2 \sqrt{3}}\left[\frac{1}{b_{1}-b_{0}}\left\|\psi^{\prime}\right\|_{2}^{2}-\left(\frac{\psi\left(b_{1}\right)-\psi\left(b_{0}\right)}{b_{1}-b_{0}}\right)^{2}\right]^{\frac{1}{2}} \\
\leq & \frac{1}{4 \sqrt{3}}(\mu-\nu)\left(b_{1}-b_{0}\right) .
\end{aligned}
$$

The above inequality is Corollary 1 (Part 1) of [12].

Corollary 2.9. If we put $\eta=\frac{b_{0}+b_{1}}{2}$ in (2.3), then following inequality holds

$$
\begin{aligned}
& \mid \psi\left(\frac{b_{0}+b_{1}}{2}\right) \int_{\alpha}^{\beta} p(u) d u+\psi\left(b_{0}\right) \int_{b_{0}}^{\alpha} p(u) d u+\psi\left(b_{1}\right) \int_{\beta}^{b_{1}} p(u) d u \\
& -\left(\frac{b_{0}+b_{1}}{2} \int_{\alpha}^{\beta} p(u) d u+b_{0} \int_{b_{0}}^{\alpha} p(u) d u+b_{1} \int_{\beta}^{b_{1}} p(u) d u-\int_{b_{0}}^{b_{1}} p(s) s d s\right) \\
& \times\left(\int_{b_{0}}^{b_{1}} p(s) \psi^{\prime}(s) d s\right)-\int_{b_{0}}^{b_{1}} p(s) \psi(s) d s \mid \\
\leq & {\left[\int_{b_{0}}^{b_{1}} \frac{K_{p}^{2}\left(\frac{b_{0}+b_{1}}{2}, s\right) d s}{p(s)}-\left(\int_{b_{0}}^{b_{1}} K_{p}\left(\frac{b_{0}+b_{1}}{2}, s\right) d s\right)^{2}\right]^{\frac{1}{2}} } \\
& \times\left[\int_{b_{0}}^{b_{1}} p(s)\left[\psi^{\prime}(s)\right]^{2} d s-\left(\int_{b_{0}}^{b_{1}} p(s) \psi^{\prime}(s) d s\right)^{\frac{1}{2}}\right]^{2} \\
\leq & \frac{1}{2}(\mu-\nu) H_{p}\left(\frac{b_{0}+b_{1}}{2}, s\right) .
\end{aligned}
$$


Remark 2.10. If we put $p(s) \equiv \frac{1}{b_{1}-b_{0}}$ in (2.14), then the bound of average midpoint and trapezoidal inequality holds

$$
\begin{aligned}
& \left|(1-h) \psi\left(\frac{b_{0}+b_{1}}{2}\right)+h \frac{\psi\left(b_{0}\right)+\psi\left(b_{1}\right)}{2}-\frac{1}{b_{1}-b_{0}} \int_{b_{0}}^{b_{1}} \psi(s) d s\right| \\
\leq & \frac{b_{1}-b_{0}}{2 \sqrt{3}} \sqrt{3 h^{2}-3 h+1}\left[\frac{1}{b_{1}-b_{0}}\left\|\psi^{\prime}\right\|_{2}-\left(\frac{\psi\left(b_{1}\right)-\psi\left(b_{0}\right)}{b_{1}-b_{0}}\right)^{2}\right]^{\frac{1}{2}} \\
\leq & \frac{(\mu-\nu)\left(b_{1}-b_{0}\right)}{4 \sqrt{3}} \sqrt{3 h^{2}-3 h+1}
\end{aligned}
$$

Remark 2.11. If we put $h=1$, then $\alpha=\beta=\frac{b_{0}+b_{1}}{2}$ in (2.14), then following inequality holds

$$
\begin{aligned}
& \mid \psi\left(b_{0}\right) \int_{b_{0}}^{\frac{b_{0}+b_{1}}{2}} p(u) d u+\psi\left(b_{1}\right) \int_{\frac{b_{0}+b_{1}}{2}}^{b_{1}} p(u) d u \\
& +\left(b_{0} \int_{b_{0}}^{\frac{b_{0}+b_{1}}{2}} p(u) d u+b_{1} \int_{\frac{b_{0}+b_{1}}{2}}^{b_{1}} p(u) d u-\int_{b_{0}}^{b_{1}} p(s) s d s\right) \\
& \quad \times\left(\int_{b_{0}}^{b_{1}} p(s) \psi^{\prime}(s) d s\right)-\int_{b_{0}}^{b_{1}} p(s) \psi(s) d s \mid \\
& \leq\left[\int_{b_{0}}^{b_{1}} \frac{K_{p}^{2}\left(\frac{b_{0}+b_{1}}{2}, s\right) d s}{p(s)}-\left(\int_{b_{0}}^{b_{1}} K_{p}\left(\frac{b_{0}+b_{1}}{2}, s\right) d s\right)^{2}\right]^{\frac{1}{2}} \\
& \quad \times\left[\int_{b_{0}}^{b_{1}} p(s)\left[\psi^{\prime}(s)\right]^{2} d s-\left(\int_{b_{0}}^{b_{1}} p(s) \psi^{\prime}(s) d s\right)^{2}\right]^{\frac{1}{2}} \\
& \leq \frac{1}{2}(\mu-\nu) H_{p}\left(\frac{b_{0}+b_{1}}{2}, s\right) .
\end{aligned}
$$

Remark 2.12. If we put $p(s) \equiv \frac{1}{b_{1}-b_{0}}$ in (2.15), then trapezoidal inequality holds as we achieve in (2.14).

Remark 2.13. If we put, $h=0$, then $\alpha=b_{0}$ and $\beta=b_{1}$ in (2.14), then weighted midpoint inequality holds

$$
\left|\psi\left(\frac{b_{0}+b_{1}}{2}\right)-\left(\frac{b_{0}+b_{1}}{2}-\int_{b_{0}}^{b_{1}} p(s) s d s\right)\left(\int_{b_{0}}^{b_{1}} p(s) \psi^{\prime}(s) d s\right)-\int_{b_{0}}^{b_{1}} p(s) \psi(s) d s\right|
$$




$$
\begin{aligned}
\leq & {\left[\int_{b_{0}}^{b_{1}} \frac{K_{p}^{2}\left(\frac{b_{0}+b_{1}}{2}, s\right) d s}{p(s)}-\left(\int_{b_{0}}^{b_{1}} K_{p}\left(\frac{b_{0}+b_{1}}{2}, s\right) d s\right)^{2}\right]^{\frac{1}{2}} } \\
& \times\left[\int_{b_{0}}^{b_{1}} p(s)\left[\psi^{\prime}(s)\right]^{2} d s-\left(\int_{b_{0}}^{b_{1}} p(s) \psi^{\prime}(s) d s\right)^{2}\right]^{\frac{1}{2}} \\
\leq & \frac{1}{2}(\mu-\nu) H_{p}\left(\frac{b_{0}+b_{1}}{2}, s\right) .
\end{aligned}
$$

The above inequality is Corollary 1 of [6].

Remark 2.14. If we put $p(s)=\frac{1}{b_{1}-b_{0}}$ in (2.16), then midpoint inequality holds

$$
\begin{aligned}
& \left|\psi\left(\frac{b_{0}+b_{1}}{2}\right)-\frac{1}{b_{1}-b_{0}} \int_{b_{0}}^{b_{1}} \psi(s) d s\right| \\
\leq & \frac{\left(b_{1}-b_{0}\right)}{2 \sqrt{3}}\left[\frac{1}{b_{1}-b_{0}}\left\|\psi^{\prime}\right\|_{2}^{2}-\left(\frac{\psi\left(b_{1}\right)-\psi\left(b_{0}\right)}{b_{1}-b_{0}}\right)^{2}\right]^{\frac{1}{2}} \\
\leq & \frac{1}{4 \sqrt{3}}(\mu-\nu)\left(b_{1}-b_{0}\right) .
\end{aligned}
$$

The above inequality is the Corollary 1 (Part 2) of [12].

Remark 2.15. If we put $h=\frac{1}{2}$, then $\alpha=\frac{3 b_{0}+b_{1}}{4}$ and $\beta=\frac{b_{0}+3 b_{1}}{4}$ in $(2.14)$, then following inequality holds

$$
\begin{aligned}
& \mid \psi\left(\frac{b_{0}+b_{1}}{2}\right) \int_{\frac{3 b_{0}+b_{1}}{4}}^{\frac{b_{0}+3 b_{1}}{4}} p(u) d u+\psi\left(b_{0}\right) \int_{b_{0}}^{\frac{3 b_{0}+b_{1}}{4}} p(u) d u+\psi\left(b_{1}\right) \int_{\frac{b_{0}+3 b_{1}}{4}}^{b_{1}} p(u) d u \\
& -\left(\frac{b_{0}+b_{1}}{2} \int_{\frac{3 b_{0}+b_{1}}{4}}^{\frac{b_{0}+3 b_{1}}{4}} p(u) d u+b_{0} \int_{b_{0}}^{\frac{3 b_{0}+b_{1}}{4}} p(u) d u+b_{1} \int_{\frac{b_{0}+3 b_{1}}{4}}^{b_{1}} p(u) d u-\int_{b_{0}}^{b_{1}} p(s) s d s\right) \\
& \times\left(\int_{b_{0}}^{b_{1}} p(s) \psi^{\prime}(s) d s\right)-\int_{b_{0}}^{b_{1}} p(s) \psi(s) d s \mid \\
& \leq\left[\int_{b_{0}}^{b_{1}} \frac{K_{p}^{2}\left(\frac{b_{0}+b_{1}}{2}, s\right) d s}{p(s)}-\left(\int_{b_{0}}^{b_{1}} K_{p}\left(\frac{b_{0}+b_{1}}{2}, s\right) d s\right)^{2}\right]^{\frac{1}{2}} \\
& \times\left[\int_{b_{0}}^{b_{1}} p(s)\left[\psi^{\prime}(s)\right]^{2} d s-\left(\int_{b_{0}}^{b_{1}} p(s) \psi^{\prime}(s) d s\right)^{\frac{1}{2}}\right. \\
& \leq \\
& \frac{1}{2}(\mu-\nu) H_{p}\left(\frac{b_{0}+b_{1}}{2}, s\right) .
\end{aligned}
$$


Remark 2.16. If we put $p(s)=\frac{1}{b_{1}-b_{0}}$ in (2.17), then the bound of average midpoint and trapezoidal inequality holds

$$
\begin{aligned}
& \left|\frac{1}{2}\left[\frac{\psi\left(b_{0}\right)+\psi\left(b_{1}\right)}{2}+\psi\left(\frac{b_{0}+b_{1}}{2}\right)\right]-\frac{1}{b_{1}-b_{0}} \int_{b_{0}}^{b_{1}} \psi(s) d s\right| \\
\leq & \frac{\left(b_{1}-b_{0}\right)}{4 \sqrt{3}}\left[\frac{1}{b_{1}-b_{0}}\left\|\psi^{\prime}\right\|_{2}^{2}-\left(\frac{\psi\left(b_{1}\right)-\psi\left(b_{0}\right)}{b_{1}-b_{0}}\right)^{2}\right]^{\frac{1}{2}} \\
\leq & \frac{1}{8 \sqrt{3}}(\mu-\nu)\left(b_{1}-b_{0}\right) .
\end{aligned}
$$

The above inequality is Corollary 1 (Part 3) of [12].

Remark 2.17. If we put, $h=\frac{1}{3}$, then $\alpha=\frac{5 b_{0}+b_{1}}{6}$ and $\beta=\frac{b_{0}+5 b_{1}}{6}$ in (2.14), then following inequality holds

$$
\begin{aligned}
\mid \psi\left(\frac{b_{0}+b_{1}}{2}\right) \int_{\frac{5 b_{0}+b_{1}}{6}}^{\frac{b_{0}+5 b_{1}}{6}} p(u) d u+\psi\left(b_{0}\right) \int_{b_{0}}^{\frac{5 b_{0}+b_{1}}{6}} p(u) d u+\psi\left(b_{1}\right) \int_{\frac{b_{0}+5 b_{1}}{6}}^{b_{1}} p(u) d u \\
\quad-\left(\frac{b_{0}+b_{1}}{2} \int_{\frac{5 b_{0}+b_{1}}{6}}^{\frac{b_{0}+5 b_{1}}{6}} p(u) d u+b_{0} \int_{b_{0}}^{\frac{5 b_{0}+b_{1}}{6}} p(u) d u+b_{1} \int_{\frac{b_{0}+5 b_{1}}{6}}^{b_{1}} p(u) d u-\int_{b_{0}}^{b_{1}} p(s) s d s\right) \\
\quad \times\left(\int_{b_{0}}^{b_{1}} p(s) \psi^{\prime}(s) d s\right)-\int_{b_{0}}^{b_{1}} p(s) \psi(s) d s \mid \\
\leq\left[\int_{b_{0}}^{b_{1}} \frac{K_{p}^{2}\left(\frac{b_{0}+b_{1}}{2}, s\right) d s}{p(s)}-\left(\int_{b_{0}}^{b_{1}} K_{p}\left(\frac{b_{0}+b_{1}}{2}, s\right) d s\right)^{2}\right]^{\frac{1}{2}} \\
\quad \times\left[\int_{b_{0}}^{b_{1}} p(s)\left[\psi^{\prime}(s)\right]^{2} d s-\left(\int_{b_{0}}^{b_{1}} p(s) \psi^{\prime}(s) d s\right)^{\frac{1}{2}}\right] \\
\leq \frac{1}{2}(\mu-\nu) H_{p}\left(\frac{b_{0}+b_{1}}{2}, s\right) .
\end{aligned}
$$

Remark 2.18. If we put $p(s)=\frac{1}{b_{1}-b_{0}}$ in (2.18), then bound of $\frac{1}{3}$ Simpson's rule holds

$$
\begin{aligned}
& \left|\frac{1}{3}\left[\frac{\psi\left(b_{0}\right)+\psi\left(b_{1}\right)}{2}+2 \psi\left(\frac{b_{0}+b_{1}}{2}\right)\right]-\frac{1}{b_{1}-b_{0}} \int_{b_{0}}^{b_{1}} \psi(s) d s\right| \\
\leq & \frac{\left(b_{1}-b_{0}\right)}{6}\left[\frac{1}{b_{1}-b_{0}}\left\|\psi^{\prime}\right\|_{2}^{2}-\left(\frac{\psi\left(b_{1}\right)-\psi\left(b_{0}\right)}{b_{1}-b_{0}}\right)^{2}\right]^{\frac{1}{2}} \\
\leq & \frac{1}{12}(\mu-\nu)\left(b_{1}-b_{0}\right) .
\end{aligned}
$$


The above inequality is Corollary 1 (Part 4) of [12].

Remark 2.19. If we put $\eta=b_{0}$ or $\eta=b_{1}$ and $p(s) \equiv \frac{1}{b_{1}-b_{0}}$ in (2.3), then trapezoidal inequality holds which is independent of the value of $h$

$$
\begin{aligned}
& \left|\frac{\psi\left(b_{0}\right)+\psi\left(b_{1}\right)}{2}-\frac{1}{b_{1}-b_{0}} \int_{b_{0}}^{b_{1}} \psi(s) d s\right| \\
\leq & \frac{b_{1}-b_{0}}{2 \sqrt{3}}\left[\frac{1}{b_{1}-b_{0}}\left\|\psi^{\prime}\right\|_{2}-\left(\frac{\psi\left(b_{1}\right)-\psi\left(b_{0}\right)}{b_{1}-b_{0}}\right)^{2}\right]^{\frac{1}{2}} \\
\leq & \frac{\left(b_{1}-b_{0}\right)(\mu-\nu)}{4 \sqrt{3}} .
\end{aligned}
$$

In Sections 3 and 4, we are going to present applications involving probability density function and numerical quadrature rules respectively.

\section{Application to probability density functions}

From [6], let $X$ be a continuous random variable having the probability density function $\psi:\left[b_{0}, b_{1}\right] \rightarrow \mathbb{R}_{+}$and the cumulative distribution function $\Psi:\left[b_{0}, b_{1}\right] \rightarrow$ $[0,1]$, i.e.,

$$
\begin{gathered}
\Psi(\eta)=\int_{b_{0}}^{\eta} \psi(s) d s, \quad \eta \in[\alpha, \beta] \subset\left[b_{0}, b_{1}\right], \\
E(X)=\int_{b_{0}}^{b_{1}} s \psi(s) d s
\end{gathered}
$$

and weighted expectation would be

$$
E_{p}(X)=\int_{b_{0}}^{b_{1}} p(s) s \psi(s) d s
$$

on the interval $\left[b_{0}, b_{1}\right]$. Then we have the following theorem.

Theorem 3.1. Let the assumptions of Theorem 2.3 be valid and if probability density function belongs to $L_{2}\left[b_{0}, b_{1}\right]$ space, then following inequality holds

$$
\begin{aligned}
& \mid \Psi(\eta) \int_{\alpha}^{\beta} p(u) d u+\int_{\beta}^{b_{1}} p(u) d u-b_{1} p\left(b_{1}\right)+E_{p}(X) \\
& -\left(\eta \int_{\alpha}^{\beta} p(u) d u+b_{0} \int_{b_{0}}^{\alpha} p(u) d u+b_{1} \int_{\beta}^{b_{1}} p(u) d u-\int_{b_{0}}^{b_{1}} p(s) s d s\right) \\
& \times\left(p\left(b_{1}\right)-\int_{b_{0}}^{b_{1}} p^{\prime}(s) \Psi(s) d s\right)+\int_{b_{0}}^{b_{1}} p^{\prime}(s) s \Psi(s) d s \mid \\
& \leq \frac{1}{2}(\mu-\nu) H_{p}(\eta, s)
\end{aligned}
$$

for all $\eta \in[\alpha, \beta]$. 
Proof. Put $\psi=\Psi$ in (2.3) and by using these two identities mention below, we get $(3.1)$,

$$
\int_{b_{0}}^{b_{1}} p(s) \Psi(s) d s=b_{1} p\left(b_{1}\right)-E_{p}(X)-\int_{b_{0}}^{b_{1}} p^{\prime}(s) s \Psi(s) d s
$$

and

$$
\int_{b_{0}}^{b_{1}} p(s) \Psi^{\prime}(s) d s=p\left(b_{1}\right)-\int_{b_{0}}^{b_{1}} p^{\prime}(s) \Psi(s) d s .
$$

Remark 3.2. Let the assumptions of Theorem 3.1 be valid, if we substitute $p(s) \equiv \frac{1}{b_{1}-b_{0}}$ in $(3.1)$, then following inequality holds

$$
\begin{aligned}
& \left|(1-h)\left[\Psi(\eta)-\frac{1}{b_{1}-b_{0}}\left(\eta-\frac{b_{0}+b_{1}}{2}\right)\right]+\frac{h}{2}-\frac{b_{1}-E(X)}{b_{1}-b_{0}}\right| \\
\leq & \frac{1}{b_{1}-b_{0}}\left[\frac{1}{12}\left(3 h^{2}-3 h+1\right)+h(1-h)\left(\eta-\frac{b_{0}+b_{1}}{2}\right)^{2}\right]^{\frac{1}{2}} \\
& \times\left[\left(b_{1}-b_{0}\right)\|\psi\|_{2}^{2}-1\right]^{\frac{1}{2}} \\
\leq & \frac{\mu-\nu}{2\left(b_{1}-b_{0}\right)}\left[\frac{1}{12}\left(3 h^{2}-3 h+1\right)+h(1-h)\left(\eta-\frac{b_{0}+b_{1}}{2}\right)^{2}\right]^{\frac{1}{2}} .
\end{aligned}
$$

\section{Applications to numerical quadrature rules}

Let $I_{n}: b_{0}=z_{0}<z_{1}<\ldots<z_{n}=b_{1}$ be a partition of the interval $\left[b_{0}, b_{1}\right]$ and let $\Delta z_{k}=z_{k+1}-z_{k}, k \in\{0,1,2, \ldots, n-1\}$. Then

$$
\int_{b_{0}}^{b_{1}} p(s) \psi(s) d s=Q_{n}\left(I_{n}, \psi, p\right)+R_{n}\left(I_{n}, \psi, p\right)
$$

where $Q_{n}\left(I_{n}, \psi, p\right)$ is a quadrature formula, define as

$$
\begin{aligned}
Q_{n}\left(I_{n}, \psi, p\right) & =\sum_{k=0}^{n-1}\left[\psi(\eta) \int_{\alpha_{k}}^{\beta_{k}} p(u) d u+\psi\left(z_{k}\right) \int_{z_{k}}^{\alpha_{k}} p(u) d u+\psi\left(z_{k+1}\right) \int_{\beta_{k}}^{z_{k+1}} p(u) d u\right. \\
& -\left(\eta_{k} \int_{\alpha_{k}}^{\beta_{k}} p(u) d u+z_{k} \int_{z_{k}}^{\alpha_{k}} p(u) d u+z_{k+1} \int_{\beta_{k}}^{z_{k+1}} p(u) d u-\int_{z_{k}}^{z_{k+1}} p(s) s d s\right) \\
& \left.\times\left(\int_{z_{k}}^{z_{k+1}} p(s) \psi^{\prime}(s) d s\right)\right]
\end{aligned}
$$

for all $\eta_{k} \in\left[z_{k}, z_{k+1}\right]$. 
Theorem 4.1. Let $\psi$ as be defined in Theorem 2.3. Then (4.1) holds where $Q_{n}\left(I_{n}, \psi, p\right)$ is given by formula (4.2) and the remainder $R_{n}\left(I_{n}, \psi, p\right)$ satisfies the estimates

$$
\left|R_{n}\left(I_{n}, \psi, p\right)\right| \leq \sum_{k=0}^{n-1} \frac{(\mu-\nu)}{2} H_{p}\left(\eta_{k}, s\right)
$$

for all $\eta_{k} \in\left[z_{k}, z_{k+1}\right]$.

Proof. Using (2.3) on $\left[z_{k}, z_{k+1}\right]$,

$$
\begin{aligned}
R_{k}\left(I_{k}, \psi, p\right) & =\int_{z_{k}}^{z_{k+1}} p(s) \psi(s) d s-\psi\left(\eta_{k}\right) \int_{\alpha_{k}}^{\beta_{k}} p(u) d u-\psi\left(z_{k}\right) \int_{z_{k}}^{\alpha_{k}} p(u) d u \\
& -\psi\left(z_{k+1}\right) \int_{\beta_{k}}^{z_{k+1}} p(u) d u+\left(\eta_{k} \int_{\alpha_{k}}^{\beta_{k}} p(u) d u+z_{k} \int_{z_{k}}^{\alpha_{k}} p(u) d u\right. \\
& \left.+z_{k+1} \int_{\beta_{k}}^{z_{k+1}} p(u) d u-\int_{z_{k}}^{z_{k+1}} p(s) s d s\right) \times\left(\int_{z_{k}}^{z_{k+1}} p(s) \psi^{\prime}(s) d s\right) .
\end{aligned}
$$

Summing over $k$ from 0 to $n-1$. This yields

$$
\begin{aligned}
R_{n}\left(I_{k}, \psi, p\right) & =\int_{b_{0}}^{b_{1}} p(s) \psi(s) d s-\sum_{k=0}^{n-1}\left[\psi\left(\eta_{k}\right) \int_{\alpha_{k}}^{\beta_{k}} p(u) d u+\psi\left(z_{k}\right) \int_{z_{k}}^{\alpha_{k}} p(u) d u\right. \\
& +\psi\left(z_{k+1}\right) \int_{\beta_{k}}^{z_{k+1}} p(u) d u-\left(\eta_{k} \int_{\alpha_{k}}^{\beta_{k}} p(u) d u+z_{k} \int_{z_{k}}^{\alpha_{k}} p(u) d u\right. \\
& \left.\left.+z_{k+1} \int_{\beta_{k}}^{z_{k+1}} p(u) d u-\int_{z_{k}}^{z_{k+1}} p(s) s d s\right) \times\left(\int_{z_{k}}^{z_{k+1}} p(s) \psi^{\prime}(s) d s\right)\right] .
\end{aligned}
$$

Applying absolute property on the above identity, we get

$$
\begin{gathered}
\left|R_{n}\left(I_{k}, \psi, p\right)\right|=\mid \int_{b_{0}}^{b_{1}} p(s) \psi(s) d s-\sum_{k=0}^{n-1}\left[\psi\left(\eta_{k}\right) \int_{\alpha_{k}}^{\beta_{k}} p(u) d u+\psi\left(z_{k}\right) \int_{z_{k}}^{\alpha_{k}} p(u) d u\right. \\
+\psi\left(z_{k+1}\right) \int_{\beta_{k}}^{z_{k+1}} p(u) d u-\left(\eta_{k} \int_{\alpha_{k}}^{\beta k} p(u) d u+z_{k} \int_{z_{k}}^{\alpha_{k}} p(u) d u\right. \\
\left.\left.+z_{k+1} \int_{\beta_{k}}^{z_{k+1}} p(u) d u-\int_{z_{k}}^{z_{k+1}} p(s) s d s\right) \times\left(\int_{z_{k}}^{z_{k+1}} p(s) \psi^{\prime}(s) d s\right)\right] \mid \\
\leq \frac{1}{2}(\mu-\nu) H_{p}\left(\eta_{k}, s\right) .
\end{gathered}
$$




\section{Conclusion}

Main objective of this article is to generalize the results of [6] and [12]. By introducing the weighted kernel as defined in [5], we have obtained generalization of Ostrowski-Grüss integral inequality for first differentiable functions in terms of weights. By using appropriate substitution we get different previously published results. At the end, we have also discussed some applications for probability density functions and numerical quadrature rules.

\section{References}

[1] Barnett, N.S., Cerone, P., Dragomir, S.S., Roumeliotis, J., Some inequalities for the dispersion of a random variable whose pdf is defined on a finite interval, J. Inequal. Pure and Appl. Math., 2(2001), no. 1, Art. 1, 18 pp.

[2] Barnett, N.S., Dragomir, S.S., Sofo, A., Better bounds for an inequality of Ostrowski type with applications, Demonstratio Math., 34(2001), no. 3, 533-542. Preprint RGMIA Res. Rep. Coll., 3(2000), no. 1, Art. 11.

[3] Dragomir, S.S., Wang, S., An inequality of Ostrowski-Grüss type and its applications to the estimation of error bounds for some special means and for some numerical quadrature rules, Comput. Math. Appl., 33(1997), 15-20.

[4] Grüss, G., Über das Maximum des absoluten Betrages von, Math. Z., 39(1935), 215-226.

[5] Imtiaz, M., Irshad, N., Khan, A.R., Generalization of weighted Ostrowski integral inequality for twice differentiable mappings, Adv. Inequal. Appl., 20(2016), Art. 20.

[6] Irshad, N., Khan, A.R., On weighted Ostrowski-Grüss inequality with applications, T.J.M.M, 10(2018), no. 1, 15-20.

[7] Matić, M., Pečarić, J.E., Ujević, N., Improvement and further generalization of some inequalities of Ostrowski-Grüss type, Comput. Math. Appl., 39(2000), no. 3/4, 161-175.

[8] Mitrinović, D.S., Pečarić, J.E., Fink, A.M., Classical and New Inequalities in Analysis, Kluwer Academic Publishers, Dordrecht, 1991.

[9] Mitrinović, D.S., Pečarić, J.E., Fink, A.M., Inequalities for Functions and their Integrals and Derivatives, Kluwer Academic, Dordrecht, 1991.

[10] Ostrowski, A.M., Über die absolutabweichung einer differentiebaren funktion von ihren integralmittelwert, Comment. Math. Helv., 10(1938), 226-227.

[11] Pachpatte, B.G., On Čebyšev-Gruss type inequalities via Pecaric's extention of the Montgomery identity, J. Inequal. Pure and Appl. Math., 7(2006), no. 1, Art. 11.

[12] Zafar, F., Mir, N.A., A generalization of Ostrowski-Grüss type inequality for first differentiable mappings, Tamsui Oxford J. Math. Sci., 26(2010), no. 1, 61-76.

Silvestru Sever Dragomir

"Victoria University"

College of Engineering and Science

PO Box 14428, Melbourne City, Australia

e-mail: sever.dragomir@vu.edu.au 
Nazia Irshad

"University of Karachi"

Department of Mathematics

University Road, Karachi-75270, Pakistan

e-mail: nazia_irshad@yahoo.com

Asif R. Khan

"University of Karachi"

Department of Mathematics

University Road, Karachi-75270, Pakistan

e-mail: asifrk@uok.edu.pk 